\title{
Marie-Claude Mahias (éd.), Construire les savoirs dans l'action. Apprentissages et enjeux sociaux en Asie du Sud
}

Paris, Éditions de l' Éditions de l'EHESS, Coll. « Puruṣārtha , 29, 2011, $293 \mathrm{p}$.

\section{André Padoux}

\section{OpenEdition}

\section{Journals}

Édition électronique

URL : http://journals.openedition.org/assr/24036

DOI : $10.4000 /$ assr.24036

ISSN : $1777-5825$

Éditeur

Éditions de l'EHESS

Édition imprimée

Date de publication : 30 décembre 2012

Pagination : 233

ISSN : 0335-5985

Référence électronique

André Padoux, « Marie-Claude Mahias (éd.), Construire les savoirs dans l'action. Apprentissages et enjeux sociaux en Asie du Sud », Archives de sciences sociales des religions [En ligne], 160 | octobredécembre 2012, mis en ligne le 18 mars 2013, consulté le 21 septembre 2020. URL : http:// journals.openedition.org/assr/24036 ; DOI : https://doi.org/10.4000/assr.24036

Ce document a été généré automatiquement le 21 septembre 2020.

(c) Archives de sciences sociales des religions 
Marie-Claude Mahias (éd.), Construire les savoirs dans l'action. Apprentissages et enjeux sociaux en Asie du Sud

Paris, Éditions de l' Éditions de l'EHESS, Coll. « Puruṣārtha , 29, 2011, $293 \mathrm{p}$.

André Padoux

\section{RÉFÉRENCE}

Marie-Claude Mahias (éd.), Construire les savoirs dans l'action. Apprentissages et enjeux sociaux en Asie du Sud, Paris, Éditions de l'EHESS, coll. «Purusārtha », 29, 2011, $293 p$. 
1 Ce volume aborde un aspect volontiers négligé ou mésestimé de la réalité de l'Inde où l'on tend souvent - suivant en cela la vision brahmane du monde et ses préjugés sociaux élitistes - à considérer les savoir-faire comme des savoirs inférieurs, comme les tours de main routiniers de simples exécutants dépourvus de véritables connaissances. Or, comme le montre Marie-Claude Mahias dans sa remarquable introduction (et comme le fait apparaître ce volume), ce sont là de véritables savoirs, mais activés, engageant la personne entière, le corps, les sens, et qui, dans leur élaboration et leur transmission, ont une dimension sociale importante. Ces savoirs gestuels, constitutifs de l'identité, sont encore nombreux aujourd'hui, bien que

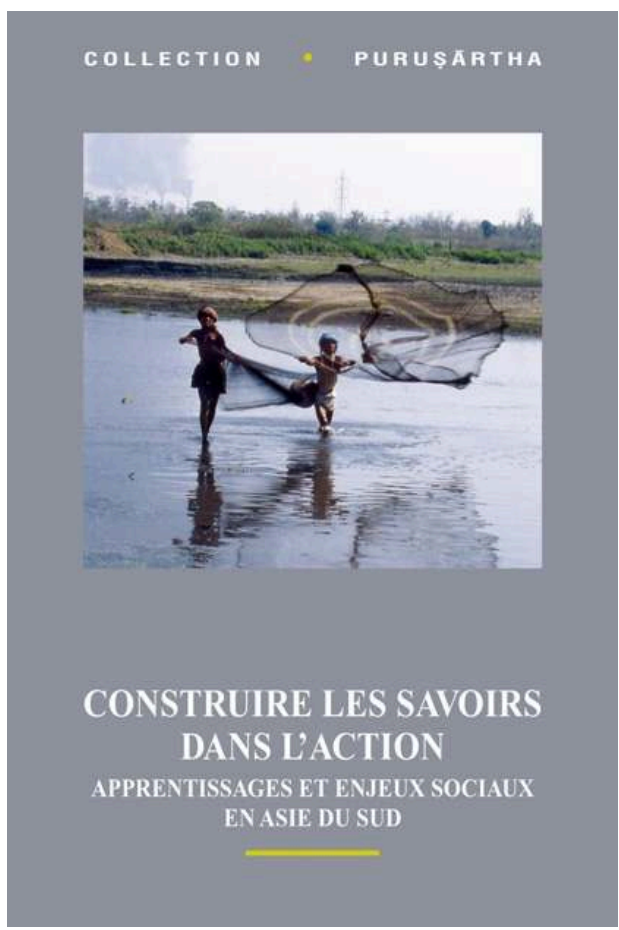
menacés par l'évolution politique et économique de l'Inde moderne. Ils offrent encore un vaste champ de recherche, qu'aborde ce recueil issu des travaux menés, en 2002-2005, par une équipe de recherche du Centre d'Études de l'Inde et de l'Asie du Sud qui s'était donné pour tâche de mettre en lumière cet aspect trop souvent occulté des activités de certains groupes sociaux indiens.

Ces études, outre leur intérêt comme descriptions de certains de ces "savoirs construits dans l'action ", ont celui d'en souligner les aspects humains et sociaux. D'abord, les modalités de l'apprentissage formant l'être, lequel, en apprenant, s'intègre à son groupe, construit sa personnalité et son identité dans ses pratiques. Celle-ci est liée à la caste. Cela parce que la caste, qui structure toujours la société, est caractérisée par la spécialisation professionnelle; mais aussi dans la mesure où, dans le monde indien, le discours du savoir, même dans le cas de groupes "inférieurs ", a toujours été celui des castes de lettrés ou dominantes - la référence aux épopées, par exemple, notamment au Rāmāyaṇa, originairement sanskrit, est répandue dans toute la population. De caractère traditionnel, souvent héréditaires, les métiers ont généralement des mythes d'origine les rattachant à une forme divine et contribuant de ce fait à les valoriser et à renforcer leur insertion dans le monde social et économique. Les articles ici rassemblés font apparaître l'importance de l'apprentissage qui, faisant appel à tous les sens, engage le corps entier et, en construisant des savoir-faire corporels, en développant une intelligence du corps, transforme tout l'être, corps et esprit.

3 Les deux premières contributions relèvent, d'ailleurs différemment, du domaine artistique. Virginie Johan («"Même l'écureuil fait ce qu'il peut.” Transmettre le savoir dans le Kūtțiyāṭam ») décrit une séance de travail d'apprentissage d'un jeune danseur dirigé par son maître dont le savoir est porté par un texte sanskrit éclairé par un manuel de jeu en langue vernaculaire. Ce que l'on voit transmis là, au cours d'une séance de deux heures, est un savoir corporel et non pas écrit d'une extrême précision. 
En effet, les attitudes, les expressions faciales et les gestes (notamment ceux des mains, les mudrās) du danseur ont tous un sens, car ils expriment - font voir - des actions, des situations dramatiques, des sentiments, traduisent même des mots. Le jeu mimétique, en l'occurrence, décrit la construction d'une hutte de feuillage par un personnage du Rāmāyaṇa. Très explicatif, cet article est éclairé par cinquante excellentes et très utiles photographies en couleur du jeune garçon (qui, comme l'écureuil, fait ce qu'il peut) et de son maître.

4 Concernant également la danse, l'étude suivante, «Savoirs dansés, enjeux statutaires dans la ronde féminine Kaikottukal i - Kerala », de Christine Guillebaud, présente une danse qui, de familiale et intime, est devenue danse de troupes se produisant sur scène, puis instituée " danse nationale du Kerala » lorsque fut créé, en 1956, cet État régional. Sont décrites trois danses exécutées dans trois contextes différents, ce qui permet, en les comparant, de faire apparaître des modifications selon qu'elles sont organisées par des associations de caste, ou par l'État lors de festivals d'art scolaires. On y voit également, non seulement le travail corporel, l'expérience corporelle, des danseuses, mais aussi les tensions entre une société de caste pour qui comptent avant tout des valeurs esthétiques et hiérarchiques et la politique d'un gouvernement désireux de diffuser les valeurs d'égalité sociale.

$5 \quad$ Les valeurs sociales traditionnelles sont également soulignées dans les études suivantes que précède une brève présentation d'Anupam Mishra (« Community planning or planning by community?») relevant les conséquences négatives de projets de développement officiels qui ignorent ou méprisent les populations locales et leurs savoir-faire traditionnels. (Attitude qui n'est d'ailleurs pas nouvelle: on pouvait déjà en voir les effets désastreux sur l'artisanat, il y a un demi-siècle.) Marie-Claude Mahias souligne ce point dans son introduction (p. 20) et le fait apparaitre clairement dans son étude sur les potières kota.

6 Sandrine Prévot (« Les chameliers du Rajasthan. Entre savoir et être ») présente le cas des Raika, important groupe de pasteurs du nord de l'Inde, dont l'identité s'inscrit dans l'histoire de leur caste, dans la hiérarchie sociale, en même temps que dans leur activité d'éleveurs de dromadaires. Celle-ci, relation entre humains et animaux, peu " outillée », suppose des savoirs efficaces et des qualités physiques et mentales très particulières. C'est, pour citer l'auteur, « un savoir-être entouré de règles sociales qui conditionnent de l'homme à l'animal ». Pénétrante, cette étude de la façon de vivre et de se vivre d'un groupe social, et d'être en rapport avec le milieu social extérieur, est attachante.

7 Diallal D. Heuzé, « Les constructeurs de bateaux de Kanpur (Inde du Nord). Savoirs de métier, culture du travail et statut », présente une petite communauté d'une même caste d'artisans charpentiers, les Nisad, qui construisent des bateaux sur le bord du Gange. Il s'y interroge, lui aussi, sur les relations entre les savoirs de métier et la conscience identitaire. Il y décrit la construction de bateaux, qui suppose une science du métier, ainsi que l'apprentissage par lequel les enfants (garçons et filles) sont précocement immergés dans le milieu du travail et, par là même, formés identitairement. Se pose aussi la question du rapport de ce groupe, avec les autres castes, hautes ou basses, vis-à-vis desquelles les Nisad s'affirment par une héroïsation de leur activité qui s'appuie sur des récits mythiques qui les ancrent dans la tradition hindoue. 
8 L'étude de Marie-Claude Mahias, «Les potières Kota (Nilgiri). Savoir-faire techniques, distinction des genres et fabrique des dieux ", est la plus longue du volume en même temps que la plus riche parce que, à la fois théorique et descriptive, elle aborde la question des savoir-faire sous plusieurs angles. Elle y montre d'abord que dès l'époque coloniale, la scolarisation, porteuse des valeurs occidentales modernes, contribua à bouleverser des acquis et des savoir-faire aussi fondamentaux que la langue, la conception de la société et du monde et même le domaine religieux. Les Kota, en effet, furent classés dans ce que la Constitution indienne de 1947 nomme les «scheduled tribes ", appellation plutôt méprisante, ces «tribus répertoriées » étant considérées comme inférieures et devant donc être protégées et aidées et notamment scolarisées pour accéder à la modernité. Or l'activité de poterie joue un rôle essentiel dans la transmission et la mise en œuvre de valeurs et de formes d'organisation sociale essentielles. Privilège des femmes, elle est un aspect central de la différence des genres et de la répartition des activités. L'auteur décrit la fabrication des pots, notamment leur tournage, faisant ainsi bien apparaître les savoir-faire, les habiletés psychomotrices, les capacités et les schèmes cognitifs qui y sont mis en œuvre. Elle rapporte également, de façon intéressante, les jugements portés en Inde sur ces opérations et qui varient selon les positions idéologiques et sociales de ceux qui s'expriment. L'examen de plusieurs projets de « valorisation » et de "développement » en montre les effets destructeurs de savoir-faire et de valeurs pourtant essentielles et donc dignes d'être protégées. Heureusement, les femmes Kota poursuivent toujours leur activité de potières et arrivent à vendre leur production tout en défendant leurs valeurs. Les photographies en couleur éclairent utilement l'aspect descriptif de cet article. Elles montrent aussi la beauté de cette activité. Il serait consternant que tout cela disparaisse, comme le font aujourd'hui tant d'activités et de pratiques de ce genre qui sont ou ont été, à leur place modeste, des éléments non négligeables de ce que l'Inde apporte à la culture du monde.

9 Mais continuons. La contribution suivante, «Extended vision. Finding fish like South Indian fishermen ", de Götz Hoeppe, examine les pratiques mises en œuvre par des pêcheurs de l'État du Kerala (Inde du Sud) pour localiser les zones où se trouvent les bancs de poissons. Ils le font, selon un savoir-faire traditionnel, en interprétant l'aspect de la mer, «la manière d'être des poissons ", comme, pour chaque équipage, la manière d'être des autres équipages.

10 L'article qui suit, «L'eau souterraine en Inde du Sud, un savoir réservé aux nouveaux maîtres de l'eau ", d'olivier Aubriot, présente un cas que l'on peut dire contraire, étudié dans les alentours de Pondichéry, où l'eau à usage agricole tend à se raréfier. Les paysans de l'endroit, qui disposent d'un savoir ancestral empirique lié à des techniques anciennes reposant notamment sur des puits et permettant de juger des ressources souterraines disponibles à peu de profondeur, tendent en effet à délaisser cette façon de voir et de faire et achètent l'eau à de riches propriétaires qui ont fait forer des puits prenant l'eau à grande profondeur dans la nappe phréatique. Ils dépendent de ce fait du savoir de ces «maîtres de l'eau » dont le statut social de dominant tient à ce qu'ils sont maîtres de l'accès à une ressource indispensable, dépendance d'autant plus grande que, interprétant les faits à la lumière de leur savoir ancestral, ils sont particulièrement soumis à des enjeux politiques, économiques ou environnementaux qui les dépassent.

11 Avec la dernière étude, "L'envers de la prise de vue. Acteurs, doublures et échafaudages gestuels dans les studios de Bombay ", d'Emmanuel Grimaud, on n'est 
plus dans le savoir-faire traditionnel, mais dans la direction d'acteurs, la gestuelle d'aujourd'hui : tableau bref, mais intéressant, par un des meilleurs connaisseurs du monde cinématographique indien.

12 Cet excellent volume prend place dignement dans la série de Purușārtha qui, depuis 1975, fait connaître des travaux de recherche poursuivis dans le cadre du CEIAS, et, par là même, beaucoup de ce qui se fait en France de plus vivant et intéressant dans le domaine de l'anthropologie de l'Inde. Il est riche d'informations et mérite entièrement d'être lu. 